\section{EVALUATIONS OF SINGLE- AND REPEATED-PLAY GAMBLES}

\author{
DOUGLas H. WEDELL \\ University of South Carolina, \\ Columbia, South Carolina
}

We encounter single instantiations of risky prospects on a regular basis, such as when we consider which car to purchase, which investment to make, or which health treatment option to choose. In each case, there is a single, unique outcome that we will experience. Regardless of whether the probability that the car we purchase will be a lemon is 1 in 10 or 5 in 10 , we will experience only one of these outcomes. So even if we pick the safe outcome ( 1 in 10) we may still get burned. At other times, we may be considering a set of repeated instantiations tied to our choice. For example, instead of purchasing a single car, one may purchase a fleet of 100 cars for a company. In such cases, it is likely that a distribution of outcomes will be observed so that 1 in 10 would translate into roughly 10 of the 100 cars in the fleet being a lemon. If we consider health decisions, the same type of distinction can be made. We may contrast the idea of choosing between risky procedures for a single person (often ourselves), or developing a choice policy applied to a population of individuals, as a health-care administrator might do.

What difference does it make to evaluate a single instantiation versus multiple instantiations of a risky prospect? Should it make a difference? This article reviews changes in decision-making behavior, which occur when evaluating single versus repeated-play gambles, beginning with a discussion of normative issues. That discussion is followed by a review of relevant behavioral studies and then a summary of theoretical explanations for these evaluations and choice patterns.

\section{NORMATIVE ISSUES}

When von Neuman and Morgenstern [1] provided a normative basis for selecting the alternative with the highest expected utility (EU), they essentially argued that a longrun perspective be applied to each unique choice set one encounters. Because the concept of expectation is based on an analysis of aggregated outcomes, EU theory naturally incorporates a long-run strategy. But, people do not always find it easy to adopt a long-run perspective when considering unique instantiations of a choice set. Instead, choices made in the short run for unique single event options and in the long run for repeated or multiple instantiations of risky prospects often differ considerably.

The Nobel prize-winning economist, Paul Samuelson, examined the distinction between short- and long-run perspectives in a brief article he wrote revolving around the choice behavior of one of his colleagues. He described how his colleague refused to risk $\$ 100$ for a $50 / 50$ chance to win $\$ 200$ [2]. Although this gamble has quite a high expected value $(E V=0.50[-\$ 100]+$ $0.50[\$ 200]=\$ 50)$, such a refusal is not problematic for EU theory, because a concave utility function could be constructed in which its EU would be less than the status quo. However, this choice became problematic when the colleague insisted he would be willing to place the bet if he were guaranteed a hundred such opportunities. Samuelson described the colleague's unwillingness to play the bet once but insistence on playing it 100 times as reflecting two different decision rules, one focused on the short run and the other on the long run, and he considered this inconsistency as irrational and a violation of EU theory.

Is it a violation of normative principles to choose differently for single and repeated risky prospects? Samuelson [2] sketched out a proof that was later fleshed out more fully

Wiley Encyclopedia of Operations Research and Management Science, edited by James J. Cochran

Copyright $\odot 2010$ John Wiley \& Sons, Inc. 
by Tversky and Bar-Hillel [3] describing when such behavior was inconsistent with EU theory. Samuelson acknowledges that refusing the single play but endorsing multiple plays can be accommodated by EU theory under some specific utility functions. However, he states that if one would refuse the single bet under all wealth levels described by the possible range of outcomes (in this case, $100[-\$ 100]=-\$ 10,000$ to $100[\$ 200]=\$ 20,000)$, then it is inconsistent to accept the repeated version. Tversky and Bar-Hillel presented a more general proof that does not depend on using EU theory as the normative standard but only depends on accepting the normative principles of transitivity and dominance. Both these proofs consider the choice of the aggregate as the same as a series of single-play choices within the range of wealth levels. However, Aloysius [4] pointed out that equating the multipleplay choice with a series of single-play choices is not a valid assumption, and he demonstrated how EU theory can be consistent with the choice pattern of Samuelson's colleague under the conditions outlined in the proofs when consideration of the aggregate is based on current one's wealth state rather than wealth states at each sequential choice point. Lopes [5,6] has also argued for the rationality of this behavior on the basis of considerations outside of the realm of EU theory. Thus, there is still a good deal of controversy concerning the normative status of switching choices between single plays of a risky prospect and multiple or repeated plays of that prospect.

\section{BEHAVIORAL FINDINGS}

Before describing behavioral results, it is useful to clarify the distributional representations of the single- and multiple-play situations, the latter being a binomial distribution of outcomes. Figure 1 presents the distributions that correspond to (i) a single play of Samuelson's bet, (ii) 10 plays of this bet, and (iii) 10 plays of the bet with payoffs reduced by a factor of 10 , equating EVs for single play and repeated plays. There are several distinctions that are immediately apparent when comparing a single play to multiple plays. As pointed out by Lopes $[5,6]$, the EV of $\$ 50$ for Samuelson's bet does not correspond to any realized outcome in the single-play situation - one walks away either $\$ 100$ poorer or $\$ 200$ richer. In contrast, the $\mathrm{EV}$ is the modal value of the distribution for the 10-play situation (in this case, there is a probability of 0.2461 of walking away with the EV). Thus, with a single-play, EV is an abstract conception that does not matchup with actual outcomes, whereas with repeated plays it is a tangible and typical result.

A second distinct feature of these distributions is that the probability of losing is quite different. With single plays, the probability of losing is simply $p=0.50$, so that half of the time one will walk away a loser. With 10 plays, one needs to lose more than twice as often as win in order to end up an
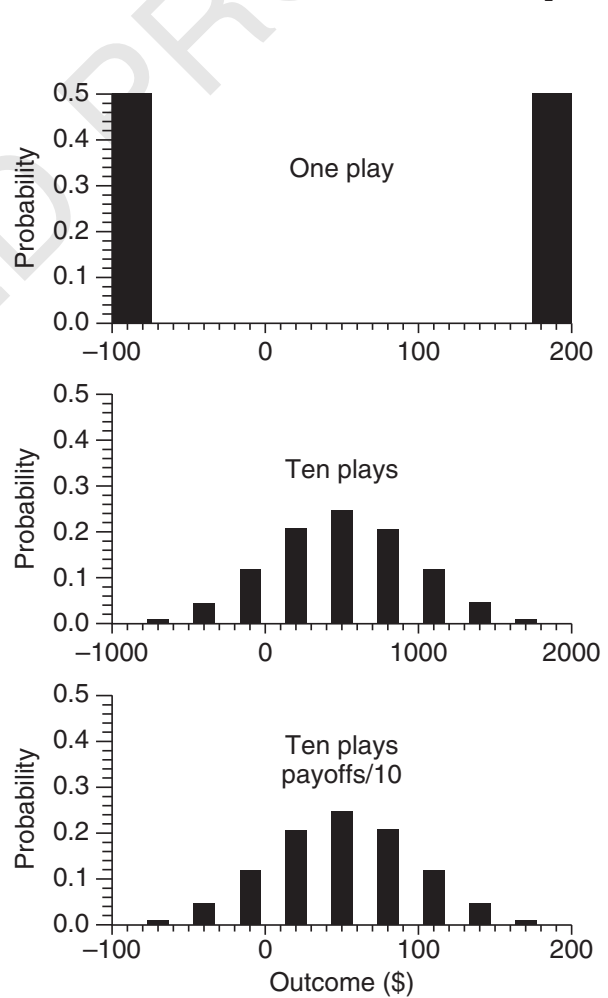

Figure 1. Outcome distributions for (a) one play of Samuelson's bet (50\% chance to lose $\$ 100$ and 50\% chance to win $\$ 200$ ), (b) 10 plays of the gamble, and (c) 10 plays of the gamble with outcomes reduced by a factor of 10 . 
overall loser, so that the probability of losing is only $p=0.17$. If the bet were played 100 times, the probability of losing would reduce to less than 1 in 2000 , radically different from the single-play situation. Of course, Samuelson [2] pointed out that the magnitude of the risk (i.e., variance of the distribution) increases with repeated plays. Thus, in the 10-play situation, one has a slight probability $(p=0.00098)$ to suffer a 10 times greater loss $(-\$ 1000)$. Because of the differences in the range of losses and the EVs for single and multiple plays of a bet, researchers have often included comparison conditions in which $\mathrm{EV}$ and range of losses are equated for the two conditions, so that the influence of these factors can be isolated. Figure 1c shows the distribution of outcomes when payoffs are reduced by a factor equal to the number of plays. Note that Samuelson's objection does not apply to this situation where risk is clearly reduced, as evidenced by the much lower variance outcomes. This type of diversification strategy is a commonly used approach for reducing risk in a portfolio of assets.

\section{Behavior for Samuelson's Bet}

Several researchers have asked people to respond to bets that parallel the one Samuelson offered his colleague in order to consider the prevalence of this pattern of responding and evaluate rationales. In a study of college students, Wedell and Böckenholt [7] found that $79 \%$ of students refused a single play of the bet, but $44 \%$ of these were willing to play the bet 100 times. Benartzi and Thaler [8] surveyed undergraduates, graduate students, and coffee shop visitors with a problem that reduced the outcomes substantially from Samuelson's gamble and found only $34 \%$ refused the single-play version. Of these, roughly $44 \%$ were willing to play the bet 100 times, a similar conditional proportion as found by Wedell and Böckenholt. Keren [9] asked the 79\% of participants, who refused to play Samuelson's bet one time, how many times it would have to be repeated before they were willing to play it. While $23 \%$ were willing to play it five times, $55 \%$ were willing to play it 100 times (a similar percentage as described above). Finally,
Redelmeier and Tversky [10] provided undergraduates with a bet similar to Samuelson's but with inflated amounts to win and lose. They found significantly fewer choices to play the bet once $(43 \%)$ than five times $(63 \%)$. In another sample, they asked participants to compare one play of the bet with five plays, five plays with six plays, and six plays with 12 plays. In each case, participants preferred the option of playing the bet more times. However, when asked whether after playing the gamble five times one would want to play it again, most participants refused, emphasizing the psychological difference between considering the set of gambles versus gamble one at a time. The results of these and other studies [11-18] indicate that the behavior of Samuelson's colleague to refuse a single play but endorse repeated plays of a gamble can be found in a substantial proportion of participants, although it may not be a majority response. This tendency may be greater for large outcome gambles, perhaps because of a higher refusal rate of the single gamble.

\section{Reducing Decision Anomalies}

It has long been known that human decision behavior often violates predictions of EU theory. To what extend is anomalous behavior found for single prospects reduced when participants evaluate repeated versions of those prospects? Keren and Wagenaar [19] investigated this question with respect to certainty and possibility effects, which Kahneman and Tversky [20] described as separable contributors to the Allais Paradox. In the certainty effect, a risky prospect A is compared to a certain (or near certain) prospect $\mathrm{B}$, and then a control pair, $\mathrm{A}^{\prime}$ and $\mathrm{B}^{\prime}$, is created by reducing the probability of winning by a constant ratio. For example, A might be (0.50 to win $\$ 250$ else $\$ 0)$ and B might be (1.00 to win $\$ 100)$. Most people prefer the certain option, $\mathrm{B}$, which means that the following inequality holds: $\mathrm{U}(\$ 100) / \mathrm{U}(\$ 250)<0.50 / 1.00$. However, reducing each probability by a factor of 5 results in options $\mathrm{A}^{\prime}(0.10$ to win $\$ 250$ else $\$ 0)$ and $B^{\prime}(0.20$ to win $\$ 100$, else $\$ 0)$. A majority of people prefer $\mathrm{A}^{\prime}$ to $\mathrm{B}^{\prime}$, which means that the following inequality holds: $\mathrm{U}(\$ 100) / \mathrm{U}(\$ 250)>0.10 / 0.20$, a contradiction 
of the choice of A over B. In the possibility effect, option probabilities are reduced by a constant ratio to create very low probability pairs that again create contradictory results. For example, one may prefer $\mathrm{C}(0.90$ to win $\$ 5000$ else $\$ 0)$ to $\mathrm{D}(0.45$ to win $\$ 12,000$ else $\$ 0$ ), but prefer $\mathrm{D}^{\prime}$ (0.01 to win $\$ 12,000$ else $\$ 0)$ to $\mathrm{C}^{\prime}$ (0.02 to win $\$ 5000$ else $\$ 0$ ).

Keren and Wagenaar [19] conducted two experiments in which participants chose between bets like those described above or the same types of bets represented as being played 10 or 100 times. For the unique, single-play gambles, they replicated both certainty and possibility effects. However, neither of these effects was significant for the repeated gambles. They concluded that different choice processes are invoked when considering the short-run unique gamble versus the long-run repeated gambles. In a follow-up, Keren [9] demonstrated a similar effect with gambles being played just once or five times. In these combined experiments, participants appeared to switch to a strategy of choosing the gamble with the higher EV when faced with multiple choices, consistent with results from Montgomery and Adelbratt [17] in which participants endorsed choosing on the basis of EV for multiple-play gambles but not for single-play gambles. The tendency for certainty and possibility effects to be reduced in the repeated-play situation implies that the choice process for repeated gambles is more consistent with EU theory than the process used for choosing single gambles.

Another violation of EU theory revolves around procedural invariance. Different preference elicitation methods can be used, such as choice, attractiveness ratings, minimum selling price, maximum buying price, and value matching. If the same utility and decision weighting processes underlie these different elicitation methods, then the preference ordering of risky prospects should be invariant across them. However, it has long been documented that preference order can be reversed by use of different methods for expressing preference [21]. In the classic response-based preference reversal, the same individual chooses between risky prospects and sets minimum selling prices for each.
One prospect is referred to as a P-bet because it has a relatively high probability to win a relatively small amount, such as a 0.90 chance to win $\$ 2$ else $\$ 0$. The other prospect is referred to as a $\$$-bet because one can win a relatively large amount but with a correspondingly smaller probability, such as a 0.10 chance to win $\$ 20$ else $\$ 0$. The typical finding is that the P-bet is preferred in choice, but the $\$$-bet is valued higher. Thus, people tend to choose the bet to which they assign a lower value.

Wedell and Böckenholt [22] examined whether the preference reversal phenomenon would obtain when gambles were represented as being played repeatedly. In both experiments, they found a significant reduction in preference reversals with an increase in the number of plays from one time to either 10 times or 100 times, although preference reversals were still significant for repeatedplay conditions. Wedell and Böckenholt reported that the majority of subjects made fewer reversals in the multiple-play condition than in the single-play condition. When prompted to explain why they changed their pricing and choice behavior, the modal response was that the perceived chances of winning changed with increase in plays. Finally, note that Haisley et al. [23] demonstrated the tendency for choice to be more in line with EV for people purchasing actual lottery tickets (singly vs in groups of five), with the repeated-play version in this case leading to significantly fewer purchases.

A third violation of EU theory arises out of considerations of sources of uncertainty as described in the Ellsberg paradox [24]. Ellsberg found that when presented with a series of choices, the majority of people opt for the gamble with a known distribution of outcomes rather than an unknown distribution, the former representing uncertainty under risk and the latter uncertainty under ambiguity. This ambiguity aversion tendency was problematic, in that it led the majority to endorse choices that violated the independence axiom in EU theory. Liu and Colman [25] investigated the possibility that ambiguity aversion is reduced in the repeated-play case, and thus the potential violation of the independence axiom is likewise reduced. In 
two experiments in which participants choose between risky and ambiguous options, they observed a strong reduction in ambiguity aversion for gambles represented as being played 100 times rather than once. These results then imply that decision makers tend to treat ambiguity and risk in a more equivalent manner when considering repeated-play gambles.

\section{Medical Decisions}

Redelmeier and Tversky [10,26] examined whether decisions of physicians would differ when considering a single instance versus aggregated instances. One study that compared aggregation across patients found that physicians were more willing to recommend a blood test when considering a single patient than a group of like patients (30\% vs $17 \%)$. In another study that compared aggregation across time, physicians were more willing to recommend a risky mediation when the term of the illness was expected to last many days than when it was to last a single day ( $42 \%$ vs $15 \%$ ), even though the chronic nature of the disease made it difficult to justify treating the single instance differently from the repeated instances. However, DeKay and Kim [11] reviewed several studies that failed to replicate results of Redelmeier and Tversky [26]. They further suggested that choice differences for single and repeated situations depend on the perceived fungibility of the outcomes. Highly fungible monetary outcomes show large differences of the manipulation, but outcomes of low perceived fungibility, such as comparison across individuals, produce little effect of the single versus repeated perspective. In summary, the results in this area are mixed, with no simple pattern being observed across studies.

\section{Investment Decisions}

Investment decisions have a natural parallel to the structure of gambles, although it is less clear about the degree to which dependencies exist among investment options. Benartzi and Thaler [8] explored short- and long-run framing in a series of experiments that used hypothetical gambles and real retirement investments. One key finding from these studies was that the format in which repeated gambles was presented was an important factor. While choices were more sensitive to EV for repeated than for single gambles, this difference was even more marked when tabular or graphic displays of the distribution of outcomes was shown (such as the displays in Fig. 1). This finding is not surprising when considering that limitations on cognitive resources would make it difficult for the average individual to accurately generate the probability distribution resulting from aggregation. (Consider an attempt at generating the outcome shown in Fig. 1b.) Benartzi and Thaler demonstrated in two additional studies that employees who were presented with the aggregate distribution over multiple years invested a much greater percentage of retirement funds in stock options than those given the one-year return rate (a $82 \%$ allocation to stocks vs a $41 \%$ allocation). Thus, when left to extrapolate consequences of repeated years of investment, people appear to be much more risk averse than when presented the resulting distribution of outcomes. Indeed, a study by Klos et al. [13] that examined investors' ability to judge various dimensions of the outcome distributions for investments and gambles found a decided advantage when the outcome distribution resulting from repeated instantiations was graphically represented.

While a number of studies have demonstrated greater acceptance of repeated or long-run investment strategies [8,12,18], Langer and Weber [14] demonstrated that the opposite effect could occur, depending on the structure of the investment. They replicated earlier work that utilized fairly large probabilities of moderate losses and then showed how gambles that included a low probability to lose a large amount were more likely to be endorsed for single plays than for repeated plays. They also demonstrated how both results were in line with prospect theory predictions.

\section{THEORETICAL EXPLANATIONS}

The clear differences in how many people respond to single and repeated instances 
of risky prospects can be explained by a variety of theoretical mechanisms. The most prominent theoretical explanation has been derived from prospect theory and hinges around loss aversion. Kahneman and Lovallo [27] referred to this result as myopic loss aversion and showed how repeated prospects represented in their aggregated form largely eliminate loss aversion tendencies. This view of the phenomena implies that single and repeated gambles are essentially processed in the same way, although the latter requires editing into aggregate form. Others have referred to this as reflecting narrow bracketing of outcomes in the short run and broad bracketing of outcomes in the long run $[18,23]$. The take home message from this approach is not that there is a change in the valuation process, but rather that there is a change in the representation upon which valuation operates. For example, if we apply a simple loss aversion weighting scheme to Samuelson's bet in which losses are multiplied by a factor of 3 , then one play of the bet is valued at $(0.5)(-100)(3)+(0.5)(200)=-50$ and so one would refuse to play it. However, applying this formulation to two plays of the bet results in a value of $0.25(-200)(3)+$ $(0.50)(100)+(0.25)(400)=0$, or indifference. According to this loss-averse weighting scheme, one would refuse a single play but choose three or more plays of the gamble. The key then is to aggregate across plays, or perhaps to see different investments as part of a broader portfolio across which one aggregates.

Other theoretical explanations focus on more distinct process differences between the situations, emphasizing shifting from qualitative to quantitative processing, a focus on aspiration levels, or on higher order features of distribution of outcomes related to variability, skewing, and rank [5-7,22,]. Lopes [5,6] pointed out that EU theory's emphasis on one moment of the outcome distribution, the mean, may not lead to the wisest decisions. Other moments related to variability and skewing may be important, especially when some outcomes are catastrophic. It is possible that individuals use a qualitative comparison of outcomes to an aspiration level before proceeding to a more systematic valuation of the prospect [22]. If this is the case, then the evaluation process would differ markedly for single- and multiple-play prospects because the multiple-play prospect would be more likely to exceed the aspiration level and proceed to a more integrative assessment. Consistent with this view are verbal reports justifying choice patterns [22] as well as evidence that risk assessment of single plays follows an additive integration pattern but that of multiple plays follows a multiplicative integration pattern [28]. Regardless of the mechanism, the extant literature is consistent with the view that people's evaluations of repeated risky prospects tend to conform more closely to normative standards, such as that described by EU theory, than their evaluations of risky prospects considered one at a time.

\section{REFERENCES}

1. Von Neuman J, Morgenstern O. Theory of games and economic behavior. Princeton, NJ: Princeton University Press; 1947.

2. Samuelson PA. Risk and uncertainty: a fallacy of large numbers. Scientia 1963;98:108-113.

3. Tversky A, Bar-Hillel M. Risk: the long and the short. J Exp Psychol: Learn Mem Cogn 1983;9:713-717. Aloysius JA. Decision making in the short and long run: repeated gambles and rationality. Br J Math Stat Psychol 2007;60:61-69.

4. Aloysius JA. Decision making in the short and long run: repeated gambles and rationality. Br J Math Stat Psychol 2007;60:61-69.

5. Lopes LL. Decision making in the short run. J Exp Psychol: Hum Learn Mem 1981;7: 377-385.

6. Lopes LL. When time is of the essence: averaging, aspiration, and the short run. Organ Behav Hum Decis Process 1996;65:179-189.

7. Wedell DH, Böckenholt U. Contemplating single versus multiple encounters of a risky prospect. Am J Psychol 1994;107:499-518.

8. Benartzi S, Thaler RH. Risk aversion or myopia? Choices in repeated gambles and retirement investments. Manage Sci 1999;45: 364-381.

9. Keren G. Additional tests of utility theory under unique and repeated condition. J Behav Decis Mak 1991;4:297-304. 
10. Redelmeier DA, Tversky A. On the framing of multiple prospects. Psychol Sci 1992;3: 191-193.

11. DeKay ML, Kim TG. When things don't add up: the role of perceived fungibility in repeated play decisions. Psychol Sci 2005;16:667-672.

12. Gneezy U, Potters J. An experiment on risk taking and evaluation periods. Q J Econ 1997; 112:631-645.

13. Klos A, Weber EU, Weber M. Investment decisions and time horizon: risk perception and risk behavior in repeated gambles. Manage Sci 2005;51:1777-1790.

14. Langer T, Weber M. Prospect theory, mental accounting and differences in aggregated and segregated evaluation of lottery portfolios. Manage Sci 2001;47:716-733.

15. Langer T, Weber M. Myopic prospect theory vs. myopic loss aversion: how general is the phenomenon?. J Econo Behav Organ 2005;56: $25-38$.

16. $\mathrm{Li} \mathrm{S}$. The role of expected value illustrated in decision-making under risk: single-play vs. multiple-play. J Risk Res 2003;6:113-124.

17. Montgomery H, Adelbratt T. Gambling decisions and information about expected value. Organ Behav Hum Perform 1982;29:39-57.

18. Thaler RH, Tversky A, Kahneman D, et al. The effect of myopia and loss aversion on risk taking: an experimental test. Q J Econ 1997; 112:647-661.

19. Keren G, Wagenaar WA. Violation of utility theory in unique and repeated gambles. J Exp Psychol: Learn Mem Cogn 1987;13:387-391.

20. Kahneman DD, Tversky A. Prospect theory: an analysis of decision under risk. Econometrica 1979;47:263-291.

21. Lichtenstein S, Slovic P. Reversal of preferences between bids and choices in gambling decisions. J Exp Psychol 1971;89:46-55.

22. Wedell DH, Böckenholt U. Moderation of preference reversals in the long run. J Exp Psychol Hum Percept Perform 1990;16:429-438.

23. Haisley E, Mostafa R, Loewenstein G. Myopic risk-seeking: the impact of narrow decision bracketing on lottery play. J Risk Uncertain 2008;37:57-75.

24. Ellsberg D. Risk ambiguity and the Savage axioms. Q J Econ 1961;75:643-649.

25. Liu HH, Colman AM. Ambiguity aversion in the long run: repeated decisions under risk and uncertainty. J Econ Psychol 2009;30: 277-284.

26. Redelmeier DA, Tversky A. The discrepancy between medical decisions for individual patients and for groups. New Engl J Med 1990;322:1162-1164.

27. Kahneman DD, Lovallo D. Timid choices and bold forecasts: a cognitive perspective on risk taking. Manage Sci 1993;39:17-31.

28. Joag SG, Mowen JC, Gentry JW. Risk perception in a simulated industrial purchasing task: the effects of single versus multi-play decisions. J Behav Decis Mak 1990;3:91-108. 


\section{Queries in Article eorms0670}

Q1. Please provide department name for the affiliation.

Q2. Please confirm if the suggested keywords are fine. 
Please note that the abstract and keywords will not be included in the printed book, but are required for the online presentation of this book which will be published on Wiley's own online publishing platform.

If the abstract and keywords are not present below, please take this opportunity to add them now.

The abstract should be a short paragraph upto 200 words in length and keywords between 5 to 10 words.

\begin{abstract}
Choices and evaluations of risky prospects often violate norms of expected utility theory and the underlying normative principles of rational decision-making. However, when risky prospects are represented as being repeatedly instantiated, choices and evaluations often change to conform more closely to the long-run perspective reflected in normative models. Differences between responses for single and repeated gambles have been analyzed normatively, explored empirically, and evaluated theoretically. Normative analyses have argued both for and against changing behavior for single and repeated gambles. Empirically, shifts in behavior for single and repeated risky prospects are found to be common, stronger when outcomes are perceived as fungible, and stronger when the repeated outcome distribution is displayed. Furthermore, these shifts have been found to occur in both laboratory and realworld settings and have been considered a possible moderator of violations of expected utility theory. A variety of theoretical explanations are posited to explain this behavior, including those that emphasize consequences of loss aversion as well as those that implicate alternative bases for evaluating single and repeated cases.
\end{abstract}

Keywords: EU theory; expected value; binomial distribution; Samuelson's bet; normative analyses

Wedell, D. H. 2011. Evaluations of Single- and Repeated-Play Gambles. Wiley Encyclopedia of Operations 